\title{
Detection of Breast Cancer using AI Techniques - A Survey
}

\author{
Riju Bhattacharya, Department of CSE, SSIPMT, Raipur. \\ Diksha Gupta ,Department of CSE, SSIPMT, Raipur. \\ Divyatara Rathod, Department of CSE, SSIPMT, Raipur.
}

\begin{abstract}
Cancer refers to any one of a large number of diseases characterized by the development of abnormal cells that divide uncontrollably and have the ability to infiltrate and destroy normal body tissue. Without treatment, it can cause serious health issues andresult in a loss of life. Breast cancer is the most common cancer among women around the world. Despite enormous medical progress, breast cancer has still remained the second leading cause of death worldwide. Early detection of cancer may reduce mortality and morbidity. This paper presents a review of the detection methods for cancer through Artificial Intelligence (AI) in different ways. Previously Microscopic reviews of tissues on glass slides are used for cancer diagnostics to improve diagnostic accuracy. We can use different techniques such as digital imaging and artificial intelligence algorithm. Cancer care is also advancing thanks to AI's ability to collect and process data. Due to the nature of processing this information, the task is often a time-consuming and tedious job for doctors. This process may be made much easier, quicker and efficient through the advancement as well as by using modified technologies.
\end{abstract}

Keywords: Breast Cancer, Artificial Intelligence (AI), Research Methodology. $* * * * *$

\section{INTRODUCTION}

Cancer is a group of diseases involving unusual cell growth with the potential to spread to other parts of the body. The correct patterns classification of breast cancer is an important real-world medical problem. Breast cancer has become one of the major causes of mortality around the world and research into cancer diagnosis and treatment has become an important issue for the scientific community[2]. Often, cancer is detected in its later stages, when it has compromised the function of one or more vital organ systems and is widespread throughout the body. Methods for the early detection of cancer are of very importance and current topic for research. Because until nownot every test is $100 \%$ accurate, it is important to understand the limitations of medical tests which are characterized by its sensitivity and specificity and false positive/false negative rates. It is generally not possible to prove what caused particular cancer because there are different reasons why there are no specific

fingerprints.

Microscopic review of tissues on glass slides is the gold stan dard for cancer diagnostics \& gives

fundamental insights into biology. Using digital images, artificial intelligence (AI) algorithms have been shown to improve diagnostic accuracy and provide quantitative metrics that cannot be provided on glass tissue slides alone. Diagnostics plays a key role throughout each patient's cancer journey-before, during and after treatment. Most cancers develop a sense of urgency for the brains behind healthcare AI research. Fortunately, researchers have found that $\mathrm{AI}$ is able to detect cancer and other diseases earlier than possible through standard diagnostic methods, which could be life-saving for most of the patients. This has been proven by studies especially focused on several types of cancer, like skin cancer and mesothelioma, which have been detected using AI with more than 95\% accuracy. AI algorithms, together with an accelerating pace of publication in a medical journal with over 500 academic publications each year featuring Artificial Neural Networks (ANNs) [6]. Previously radiologists make a relatively subjective determination for many terms from the lexicon related to a breast cancer diagnosis. Lobulation and microlobulation of nodules are two important features in the ACR lexicon. In medical and other domains, linear programming approaches were efficient and effective methods [7, 12, 13, 14]. Recently, intelligent methods such as NN and support vector machines have been intensively used for classification tasks [15]. Feedforward neural networks are a basic type of ANNs capable of approximating generic classes of functions, including continuous and integrable ones. An important class of feedforward neural networks is MLPNNs. The MLPNNs have features such as the ability to learn and generalize, smaller training set requirements, fast operation, ease of implementation and therefore they are the most commonly used neural network architectures $[8,9,10]$.

\section{Breast Cancer}

Breast cancer is a malignant tumor that starts in the tissues of the breast. This cancer can expand directly into surrounding areas or maybe distribute to distant parts of the body. The disease occurs almost exclusively in females, but men can also develop this type of cancer [4].Breast cancer is the most common cancer and the second leading cause of 
death among women around the world. Breast cancer occurs when the cell tissues of the breast become abnormal and uncontrollably divided. These abnormal cells form a large lump of tissues, which consequently becomes a tumor [1]. Although scientists know some of the risk factors (i.e. aging, genetic risk factors, family history, menstrual periods, not having children, obesity) that increase a woman's chance of developing breast cancer, they do not yet know what causes most breast cancers or exactly how some of these risk factors cause cells to become cancerous. Research is underway to learn more and scientists are making great progress in understanding how certain changes in DNA can cause normal breast cells to become cancerous [2,4]. Fuzzy Logic using mammograms provides the best classifier technique for the detection and classification of breast cancer, yielding an accuracy of $99.73 \%$. The disadvantages of FL are generally the numerous probable fuzzy rules that exponentially increase with the dimensionality of pattern space (R. Jain \& Abraham, 2004)[] and the lack of a systematic method for fuzzy system design. However, the method is easy to understand. The approach is simple and suitable for problems that do not require high accuracy [4].

\section{Artificial Intelligence (AI)}

AI techniques are used to produce and develop computer software programs. AI is an application that can re-create human perception. This application generally requires to obtain input to provide AI with analysis or dilemma solving, as well as the ability to categorize and identify objects [4].

However, manual classification of images is a challenging and time-consuming task, being highly susceptible to interobserver variability and human errors, resulting in extremely poor and critical outcomes, thus leads to increase the workload of radiologists because of their significant shortage. In addition, medical care costs for imaging are rapidly increasing. Therefore, new methods for diagnosis are required.

\section{Research Methodology}

\section{A. Image processing:}

Medical imaging has become an important part of the treatment methods for detection of earlier diagnosis through the years. Medical imaging is the first step towards the detection and prevention of cancer in many cases by avoiding the spread of cancer and assists in the treatment or total elimination of cancer. MRI, CT imaging, ultrasound (US) imaging, X-ray imaging, mammography and so on, are typical imaging modalities used for fighting cancer [4]. Medical imaging techniques have widely been in use in the diagnosis and detection of breast cancer. The drawback of applying these techniques is the large time consumption in the manual diagnosis of each image pattern by a professional radiologist. Automated classifiers could substantially upgrade the diagnosis process, in terms of both accuracy and time requirement by distinguishing benign and malignant patterns automatically[1].Different AI algorithms are used to detect and classify different types of cancers. The AI feed-forward ANN algorithm by using backpropagation for enhancing the image processing mechanism to identify the cancer symptoms

\section{B. Microscope:}

The modified standard optical microscope to have built-in real-time AI capabilities [5]. The integration of modern AI into a standard microscope that is routinely used to review cancer specimens and perform basic and translational research [5]. This required integrating an augmented reality display into the optics, training a deep neural network for cancer detection integration of projection optics into an existing microscope provided a parallax-free, highresolution display of information derived by machine learning overlaying the sample. The AI algorithm was running in real-time using the live image feed from the camera and was generating high accuracy cancer detection results. The augmented microscope has applications in research and clinical usage and could provide complementary technology to digital pathology based on whole slide imagery. Its main promise lies in expanding the availability of modern AI algorithms [5].

\section{ArtificialNeural Network}

An artificial neural network (ANN) or commonly as Neural Network (NN) is an interconnected group of artificial neurons that uses a mathematical or computational model for information processing. Artificial neural networks (ANN) have found extensive deployment in a broad spectrum of assortment, perception, association and control applications. That is based on structures and functions of biological neural networks. Whereas, the structure of the ANN-based on the flow of information.Non-linear statistical data. That means complex relationship defines between input and output. As a result, we found different patterns. Also, we call the ANN as a neural network ANN composed of multiple nodes. That imitate biological neurons of the human brain. Although, we connect these neurons by links. Also, they interact with each other. Although, nodes are used to take input data. Further, perform simple operations on the data. As a result, these operations are passed to other neurons.

An ANN is a mathematical representation of theHuman neural architecture, reflecting its "learning" and "generalization" abilities. For this reason, ANNs belong to the field of artificial intelligence. ANNs are widely applied in research because they can model highly non-linear systems in which the relationship among the variables is 
unknown or very complex. A review of various classes of neural networks can be

Found $[16,17]$.

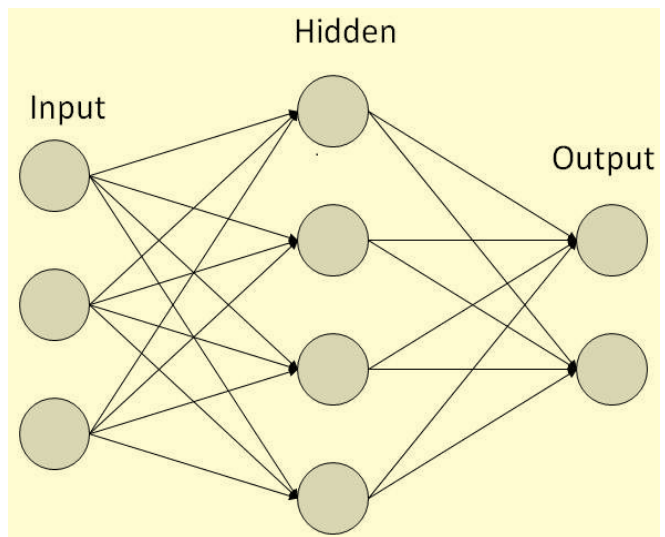

Fig 1: Artificial Neural Network ANN

\section{Mammography}

A mammogram is an $\mathrm{x}$-ray picture of the breast. It can be used to check for breast cancer in women who have no signs or symptoms of the disease. It can also be used if you have a lump or other sign of breast cancer. Screening mammography is the type of mammogram that checks you when you have no symptoms. With recent functions involving examinations, considerable interest with regard to the utilization of computational strategies to assist detection and diagnosis of breast cancer among most cancers is concentrated on mammography. The particular mammography technique is a simple yet effective tool for prognosis that involves breast cancer at an earlier stage [19].

\section{E. Feedforward Neural Network}

This neural network is one of the examples of the simplest form of ANN, where the data or the input propagates in one direction. The data flows through the input nodes and exit on the output nodes. This neural network may or may not have the hidden layers. In simple words, it is generally using a classified activation function in front wave and usually no back spread. Below is a Single layer feed forward network. Thus, the sum of the products of inputs and weights are calculated and send to the output.

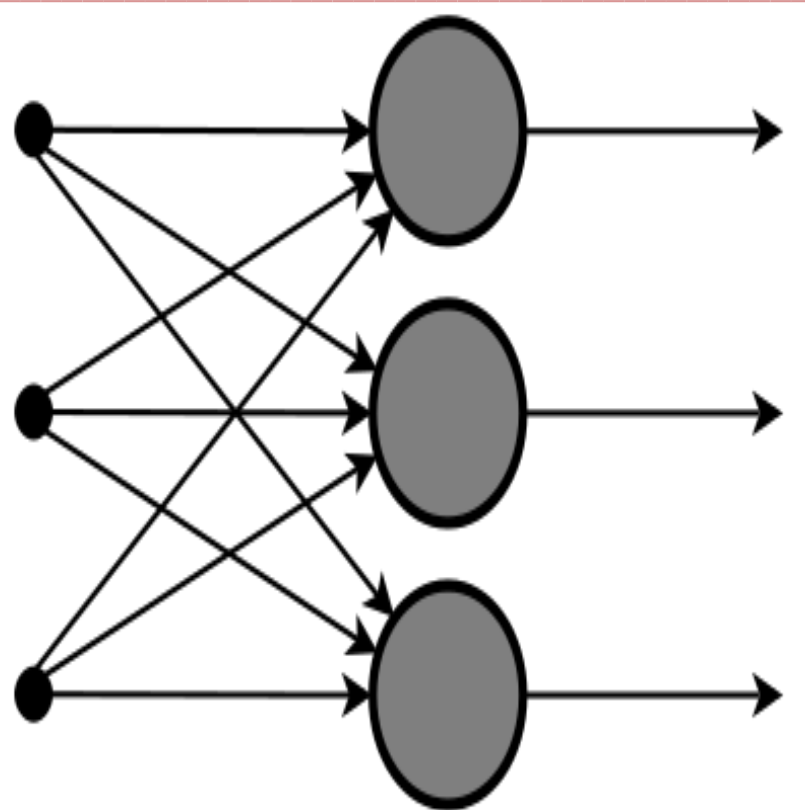

Fig 2: Information flow in ANN

Application of Feedforward neural networks are found in computer vision and speech recognition was classifying the target classes are complicated. These kind of Neural Networks are responsive to noisy data and easily maintain. The X-Ray image is a process of overlaying two or more images based on the fusion edges. In this network flow of information is accidental. A unit which is used to send information to another unit that does not receive any information. Also, no feedback loops are present in this. Although, used in recognition of a pattern. As they contain fixed inputs and outputs.

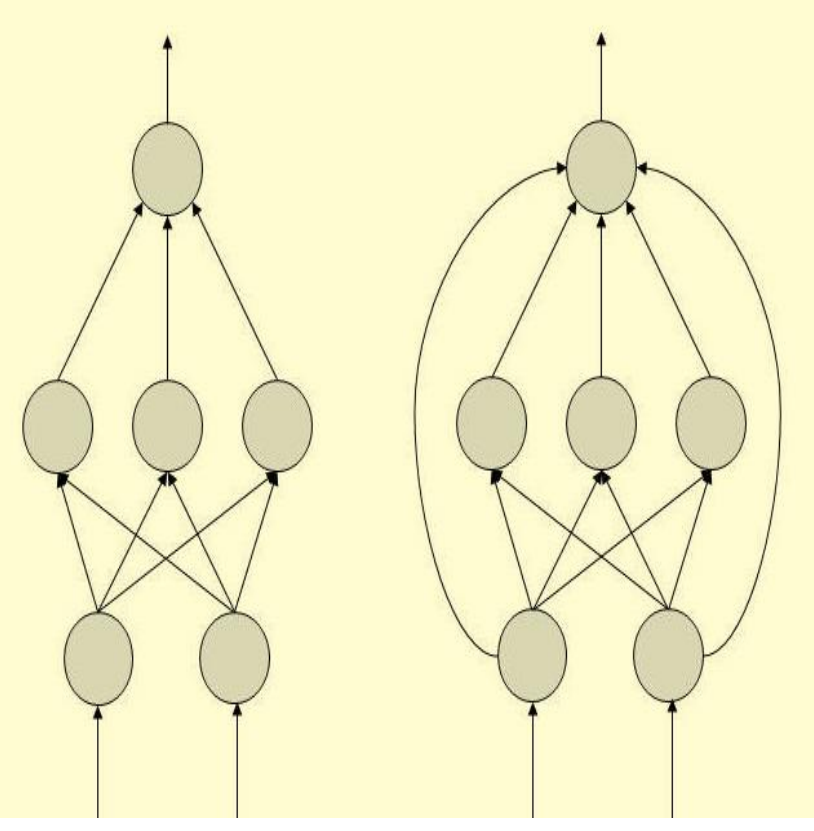

Fig 3: Type of Artificial Neural Network Feed Forward NN 


\section{F.} Fuzzy systems

Fuzzy logic was invented by Zadeh9 in 1965 for handling uncertain and imprecise knowledge in real-world applications. (R. Jain \& Abraham, 2004) applied rule generation based on homogeneous fuzzy sets and used another rule mean standard deviation of attribute values. (Al-Daoud, 2010) proposed a modified fuzzy c-means

RBF network, and (Soria et al., 2013) adopted a fuzzy algorithm linguistic rule. (GÖRGEL, SERTBAŞ, \& UÇAN, 2012) applied fuzzy subtractive ANFIS. (Onan, 2015) presented a hybrid intelligent fuzzy-rough nearest neighbor algorithm. All of these researchers used different fuzzy rules on mammogram data and obtained a high accuracy (>90\%) in detecting and classifying breast cancer. (R. Jain \& Abraham, 2004) used rule generation based on a histogram of attribute values, and (Gerald Schaefer et al., 2007) proposed a system for breast cancer detection on thermography by using a fuzzy classification system (Miranda \& Felipe, 2014) presenting fuzzy omega algorithm method. Jain, Schaefer, and Miranda obtained good accuracy that exceeded $80 \%$ by using different fuzzy rules. (R. Jain \& Abraham, 2004) applied a generation rule that depended on the fuzzy partition of overlapping areas and obtained a low accuracy of $62.57 \%$.

\section{G. MicroRNA System}

MicroRNAs (miRNAs) are a class of single-stranded small non-coding RNA molecules of $\sim 22$ nucleotides. A large microRNA (miRNA) expression dataset, with the goal of optimizing miRNA biomarker selection using neural network cascade (NNC) modeling. Our results identified numerous candidate miRNA biomarkers that are technically suitable for BC detection. We combined three miRNAs (miR-1246, miR-6756-5p, and miR-8073) into a single panel to generate an NNC model, which successfully detected BC with $97.1 \%$ accuracy in an independent validation cohort comprising 429 BC patients and 895 healthy controls[20].

\section{Result \& Discussion}

In our study, we reviewed many technologies and method used for the detection of cancer, especially breast cancer by using artificial intelligence in different fields like digital imaging, mammography, microscopes, ANN using feed forward algorithm as well as fuzzy logic system. Some methodology shows a high percentage of accuracy for cancer detection. Although the diagnosis of breast cancer can be highly accurate, it is not necessarily the same as the results obtained in other sets of images. Lots of new technologies are introducing for the enhancement in the cancer diagnosis.

\section{Conclusion}

The purpose of this review is to analyze the contents of recently published literature with special attention to techniques and states of the art of artificial intelligence in cancer detection. Thus Artificial Intelligence enhances as well as also diagnose cancer more accurately and precisely. Thus making the cancer diagnoses test result to be more accurate and also rapid so that the person diagnosed with cancer starting their treatment as soon as possible.

[1]. Sadoughi, Farahnaz, Zahra Kazemy, Farahnaz Hamedan, Leila Owji, Meysam Rahmanikatigari, and Tahere Talebi Azadboni. "Artificial intelligence methods for the diagnosis of breast cancer by image processing: a review." Breast Cancer: Targets and Therapy 10 (2018): 219.

[2]. Marcano-Cedeño, Alexis, Joel Quintanilla-Domínguez, and Diego Andina. "WBCD breast cancer database classification applying artificial metaplasticity neural network." Expert Systems with Applications 38, no. 8 (2011): 9573-9579.

[3]. Krittanawong, C. "The rise of artificial intelligence and the uncertain future for physicians." European journal of internal medicine 48 (2018): e13-e14.

[4]. Al-shamasneh, Alaá Rateb Mahmoud, and Unaizah Hanum Binti Obaidellah. "Artificial intelligence techniques for cancer detection and classification: review study." European Scientific Journal, ESJ 13, no. 3 (2017).

[5]. Chen, Po-Hsuan Cameron, Krishna Gadepalli, Robert MacDonald, Yun Liu, Kunal Nagpal, Timo Kohlberger, Jeffrey Dean, Greg S. Corrado, Jason D. Hipp, and Martin C. Stumpe. "Microscope 2.0: An Augmented Reality Microscope with Real-time Artificial Intelligence Integration." arXiv preprint arXiv:1812.00825 (2018).

[6]. Gant, Vanya, Susan Rodway, and Jeremy Wyatt. "Artificial neural networks: practical considerations for clinical applications." Clinical applications of artificial neural networks(2001): 329-356

[7]. Bennett, Kristin P., and Olvi L. Mangasarian. "Robust linear programming discrimination of two linearly inseparable sets." Optimization methods and software 1, no. 1 (1992): 23-34.

[8]. Basheer, Imad A., and Maha Hajmeer. "Artificial neural networks: fundamentals, computing, design, and application." Journal of microbiological methods 43, no. 1 (2000): 3-31.

[9]. Chaudhuri, B. B., and Ujjwal Bhattacharya. "Efficient training and improved performance of multilayer perceptron in pattern classification." Neurocomputing 34, no. 1-4 (2000): 11-27.

[10]. Haykin, Simon. Neural networks: a comprehensive foundation. Prentice Hall PTR, 1994.

[11].Bennett, Kristin P., and Olvi L. Mangasarian. "Robust linear programming discrimination of two linearly inseparable sets." Optimization methods and software 1, no. 1 (1992): 23-34.

[12].Freed, Ned, and Fred Glover. "Applications and implementation: A linear programming approach to the 
discriminant problem." Decision Sciences 12, no. 1 (1981): 68-74.

[13]. Grinold, Richard C. "Mathematical programming methods of pattern classification." Management science 19, no. 3 (1972): 272-289.

[14].Smith, Fred W. "Pattern classifier design by linear programming." IEEE transactions on computers 100, no. 4 (1968): 367-372.

[15].Ryu, Young U., Ramaswamy Chandrasekaran, and Varghese S. Jacob. "Breast cancer prediction using the isotonic separation technique." European Journal of Operational Research 181, no. 2 (2007): 842-854.

[16].Zupan, Jure, Johann Gasteiger, and Jure Zupan. "Neural networks in chemistry and drug design." (1999): 2011.

[17]. Aleksander I, Morton H. An introduction to neural computing. Int Thomson Comput Press, London 1995.

[18].Jain, Ravi, and Ajith Abraham. "A comparative study of fuzzy classification methods on breast cancer data." Australasian Physics \& Engineering Sciences in Medicine 27, no. 4 (2004): 213-218.

[19]. Cheng, Heng-Da, Juan Shan, Wen Ju, Yanhui Guo, and Ling Zhang. "Automated breast cancer detection and classification using ultrasound images: A survey." Pattern recognition 43, no. 1 (2010): 299-317.

[20].Cui, Xiaomeng, Zhangming Li, Yilei Zhao, Anqi Song, Yunbo Shi, Xin Hai, and Wenliang Zhu. "Breast cancer identification via modeling of peripherally circulating miRNAs." PeerJ 6 (2018): e4551. 